\title{
Interdiffusion of molecular acceptors through organic layers to metal substrates mimics doping-related energy level shifts
}

\author{
Steffen Duhm, ${ }^{1, a)}$ Ingo Salzmann, ${ }^{1}$ Benjamin Bröker, ${ }^{1}$ Hendrik Glowatzki, ${ }^{1}$ \\ Robert L. Johnson, ${ }^{2}$ and Norbert Koch ${ }^{1, b)}$ \\ ${ }^{1}$ Institut für Physik, Humboldt-Universität zu Berlin, Newtonstr. 15, D-12489 Berlin, Germany \\ ${ }^{2}$ Institut für Experimentalphysik, Universität Hamburg, Luruper Chaussee 149, D-22761 Hamburg, Germany
}

(Received 22 May 2009; accepted 8 August 2009; published online 3 September 2009)

\begin{abstract}
Photoemission measurements reveal energy level shifts toward the Fermi level when a strong electron acceptor (tetrafluoro-tetracyanoquinodimethane, F4-TCNQ) is deposited on pristine layers of $4,4^{\prime}, 4^{\prime \prime}$-tris $(N, N$-diphenyl-amino)triphenylamine (TDATA) or 4,4'-bis $(N$-carbazolyl)biphenyl (CBP). The shifts of the TDATA and CBP energy levels toward the Fermi level of the Au substrate could, in principle, arise from $p$-type doping of the intrinsic organic layers. While this indeed takes place in TDATA, doping of CBP by F4-TCNQ, i.e., charge transfer complex formation, does not occur. The shifts observed in CBP arise from the diffusion of F4-TCNQ toward the Au substrate, which modifies the buried metal surface potential, leading to a realignment of the energy levels of the organic overlayer. (C) 2009 American Institute of Physics. [DOI: 10.1063/1.3213547]
\end{abstract}

Strong electron acceptor molecules are frequently used for electrical doping of hole transport layers in organic electronic devices. ${ }^{1-4}$ They yield improved device performance due to organic/organic $(\mathrm{O} / \mathrm{O})$ charge transfer complex (CTC) formation in the codeposited thin films, which increases the layer conductivity and lowers the hole injection barrier (HIB; defined as energy difference between $E_{F}$ and the highest occupied molecular orbital onset) at the anode ( $p$-type doping). Strong electron acceptors can also be used to precisely adjust the energy levels at organic/metal $(\mathrm{O} / \mathrm{M})$ interfaces, ${ }^{5,6}$ even in the absence of doping. In this case, an O/M CTC (Ref. 7) is formed, which increases the effective substrate surface potential and thus reduces the HIB for any subsequently deposited organic material. ${ }^{5,6}$ Note that HIB lowering due to O/M CTC formation may be misinterpreted as $p$-type doping of the organic layer $(\mathrm{O} / \mathrm{O}$ CTC) because a shift of the donor energy levels toward the substrate Fermi level $\left(E_{F}\right)$ occurs in both cases. Acceptors are often small molecules like tetrafluoro-tetracyanoquinodimethane (F4-TCNQ) [chemical structure shown in Fig. 1(a)], which can readily diffuse throughout crystalline organic layers, ${ }^{8}$ furthermore, diffusion through amorphous organic layers may also occur, particularly when intermolecular interactions are weak. In such cases, the acceptor may reach the metal substrate [Fig. 1(b)] via diffusion and may form O/M CTCs. It may thus be difficult to identify the underlying mechanism that results in observed energy level shifts for organic donor/acceptor heterosystems, as O/M CTC formation may mimic $p$-type doping.

To clarify the role of acceptor interdiffusion on the energy levels in organic heterosystems, we used ultraviolet photoelectron spectroscopy (UPS) to investigate the combination of F4-TCNQ with the donor $4,4^{\prime}, 4^{\prime \prime}$-tris $(N, N$-diphenyl-amino)triphenylamine (TDATA) and $4,4^{\prime}$-di $(N$-carbazolyl)biphenyl (CBP) [Fig. $1(\mathrm{a})$ ], respectively, on Au substrates. While TDATA can be doped with

\footnotetext{
${ }^{a}$ Present address: Graduate School of Advanced Integration Science, Chiba University, Chiba, Japan. Electronic mail: duhm@restaff.chiba-u.jp.

${ }^{b)}$ Electronic mail: norbert.koch@physik.hu-berlin.de.
}

F4-TCNQ, ${ }^{9,10}$ the ionization energy of CBP with $6.3 \mathrm{eV}$ (Ref. 11) is too large to allow for CTC formation with F4TCNQ. Both TDATA and CBP form amorphous films, ${ }^{12}$ which rules out possible influences of film structure on our results. Therefore, the effects of acceptor diffusion on interface energetics could be studied in the presence (with TDATA) and absence (with CBP) of O/O CTCs.

Photoemission experiments were performed at the FLIPPER II end station (base pressure $1 \times 10^{-10} \mathrm{mbar}$ ) at HASYLAB (Hamburg, Germany). ${ }^{13}$ Au substrates were freshly deposited $\sim 600 \AA \mathrm{Au}$ films on $\mathrm{Cu}$-foil cleaned by Ar-ion sputtering. F4-TCNQ (Fluka), CBP (Aldrich), and TDATA (Sensient) were thermally evaporated, at deposition rates of about $1 \AA / m i n$. The film mass thickness was monitored with a quartz crystal microbalance. The photon energy was $22 \mathrm{eV}$ and the energy resolution $200 \mathrm{meV}$. The secondary electron cutoffs [for determination of the sample work function $(\phi)$ ] were measured with the sample biased at $-3.00 \mathrm{~V}$. The error of binding energy (BE) and work function values is \pm 0.10 (a)
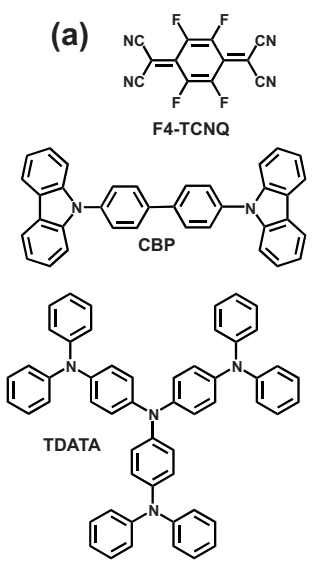

(b)

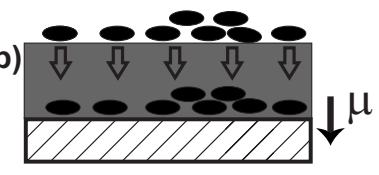

(c)

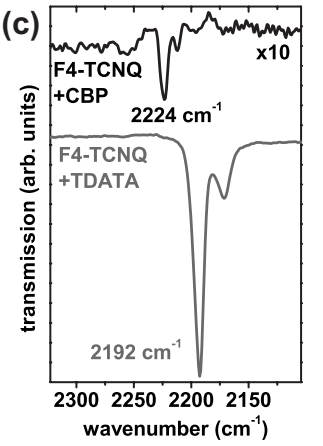

FIG. 1. (a) Chemical structure of F4-TCNQ, CBP, and TDATA. (b) Scheme of interdiffusion of small acceptor molecules through an organic thin film to the metal substrate and acceptor/metal dipole layer $(\mu)$ formation. (c) IR absorption spectra of $200 \AA$ thick films of TDATA and CBP on $\mathrm{SiO}_{x}$, both codeposited with F4-TCNQ. 

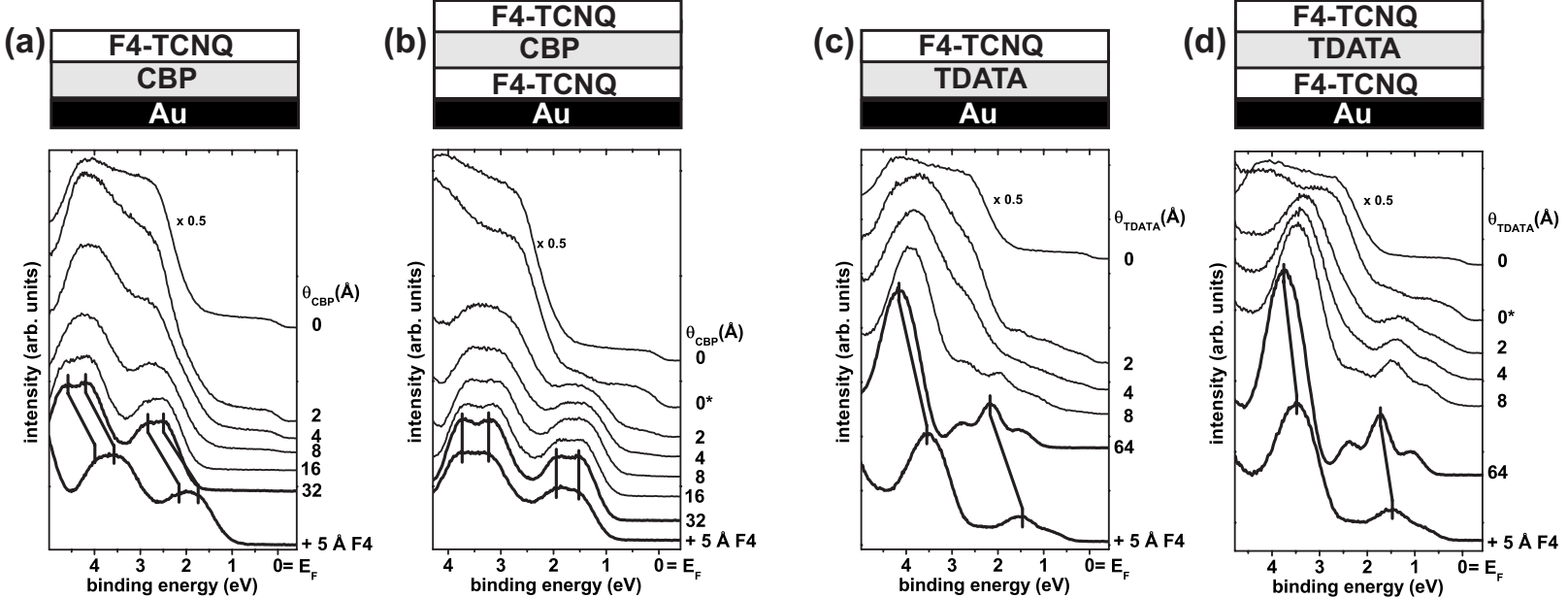

FIG. 2. Thickness-dependent UPS spectra of CBP and TDATA on Au (a) and (c) and on $5 \AA$ F4-TCNQ/Au (b) and (d), in each case with a $5 \AA$ thick F4-TCNQ top-layer. The deposition sequence (not the actual sample composition) is sketched in the upper part of the figure. The shifts of the molecular features caused by F4-TCNQ top-deposition are marked by lines. $\theta_{i}$ denotes the layer thickness of CBP or TDATA, $0^{*}$ the $5 \AA$ F4-TCNQ/Au layer, $E_{F}$ Fermi-energy and F4 F4-TCNQ.

eV. The time between evaporation and measurement was typically $10 \mathrm{~min}$. We collected spectra repeatedly within ca. $1 \mathrm{~h}$ after each evaporation step. On that time scale, no shifts were observed. Infrared (IR) absorption measurements were done on $200 \AA$ thick codeposited films on $\mathrm{SiO}_{x}$ (F4-TCNQ + CBP and F4-TCNQ+TDATA with a molecular ratio of $1: 1)$. These films were vacuum sublimed in high vacuum at evaporation rates of $\sim 5 \AA / \mathrm{min}$. IR spectra were obtained using a Fourier-transform-IR spectrometer IFS 66v/S (Bruker). All preparation steps and measurements were performed at room temperature.

The IR spectra of the C-N stretching modes of F4TCNQ in Fig. 1(c) clearly indicate O/O CTC formation of F4-TCNQ with TDATA and its absence with CBP. Particularly, the $b_{1 u}$ mode at $2227 \mathrm{~cm}^{-1}$ for crystalline neutral F4-TCNQ (Ref. 14) is a clear indicator of the degree of charge transfer in CTCs. ${ }^{15}$ The peak at $2224 \mathrm{~cm}^{-1}$ in the F4-TCNQ+CBP film results from F4-TCNQ in the neutral state; the shift of $3 \mathrm{~cm}^{-1}$ compared to crystalline F4-TCNQ arises from the different intermolecular interaction for the acceptor in the inert CBP matrix compared to the crystalline structure. For the F4-TCNQ+TDATA film, this peak was shifted to $2192 \mathrm{~cm}^{-1}$, demonstrating the formation of $\mathrm{O} / \mathrm{O}$ CTC with close to one electron charge transfer. ${ }^{14,15}$

In the UPS spectra, the deposition of CBP on pristine Au attenuated the Au photoemission features and four moleculederived photoemission features appeared between 1 and $5 \mathrm{eV}$ BE [Fig. 2(a)]. From 2 to $32 \AA \mathrm{CBP}$ coverage, the HIB increased from 1.40 to $1.80 \mathrm{eV}$ (Fig. 3) due to reduced metal substrate screening at larger thicknesses ${ }^{16,17}$ and possible changes of molecular conformation between monolayer and multilayer. ${ }^{18}$ For coverages $>8 \AA$ the intensity at the metal Fermi-edge was totally suppressed, i.e., the entire substrate was covered with $\mathrm{CBP}$; therefore, it is most likely that no pinholes in the amorphous CBP film of $32 \AA$ coverage are present. The sample work function decreased from 5.20 (pristine $\mathrm{Au})$ to $4.50 \mathrm{eV}(2 \AA \mathrm{CBP} / \mathrm{Au})$ (Fig. 3) due to the electron push back effect ${ }^{16,17}$ and remained constant for higher CBP coverages. These findings are in agreement with previous reports on CBP/Au. ${ }^{11}$ The deposition of $5 \AA$ F4TCNQ on top of the thick CBP layer increased $\phi$ by $0.70 \mathrm{eV}$, shifted all of the molecule-derived features in the valence electron region rigidly toward $E_{F}$ by $0.75 \mathrm{eV}$ [Figs. 2(a) and $3]$, and the spectral features were slightly broadened (by ca. $0.1 \mathrm{eV})$.

The deposition of $5 \AA$ F4-TCNQ on Au resulted in two significant O/M CTC-peaks centered at 0.45 and $1.50 \mathrm{eV}$ $\mathrm{BE}$, and $\phi$ was increased by $0.35 \mathrm{eV}$ [Figs. 2(b) and 3], as expected from previous studies. ${ }^{5}$ The energy levels of CBP on F4-TCNQ/Au were aligned to the modified substrate work function, i.e., the CBP features were found at $0.90 \mathrm{eV}$ lower $\mathrm{BE}$ in comparison to $\mathrm{CBP} /$ pristine $\mathrm{Au}$. The decrease in $\phi$ by $2 \AA \mathrm{CBP}$ adsorption was $0.40 \mathrm{eV}$ and $\phi$ was remained essentially constant at larger CBP coverages. With increasing CBP layer thickness, the HIB shifted from 0.70 to $0.90 \mathrm{eV}$ (Fig. 3). Subsequent deposition of $5 \AA$ F4-TCNQ on top of the CBP multilayer did not result in any significant changes in the spectrum, apart from a slight broadening $(<0.1 \mathrm{eV})$.

When TDATA was deposited on pristine Au, a coverage of $8 \AA$ was sufficient to suppress the photoemission intensity at the metal Fermi-edge [Fig. 2(c)]. The work function decreased from 5.10 (Au) to $3.90 \mathrm{eV}$ (4 ̊ TDATA/Au) (Fig. 3). With increasing TDATA coverage, the HIB increased from 0.30 to $1.00 \mathrm{eV}$ and $\phi$ decreased further from 3.90 (4 TDATA/Au) to $3.65 \mathrm{eV}$ (64 ̊ TDATA/Au). The significant
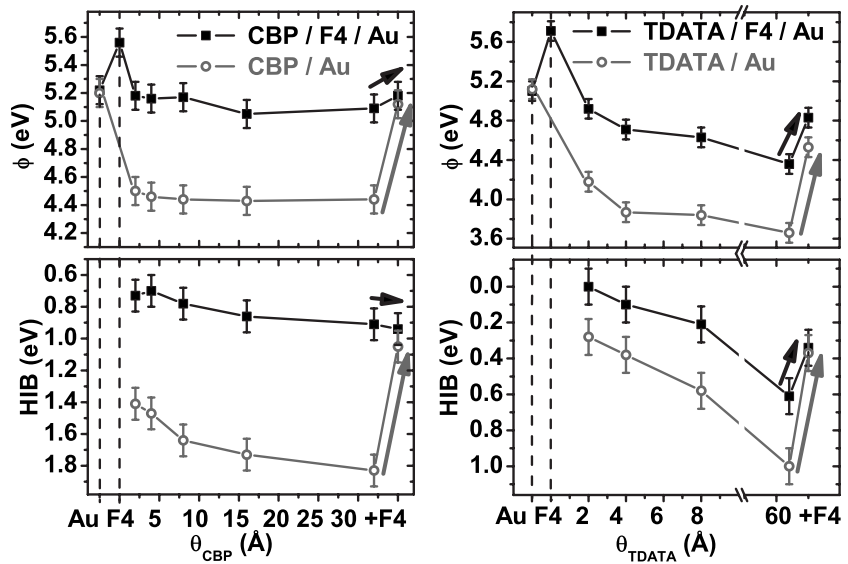

FIG. 3. Work function $(\phi)$ and HIB of CBP and TDATA on pristine Au and on $5 \AA$ F4-TCNQ precovered Au as a function of overlayer thickness $(\theta)$. The impact of $5 \AA$ F4-TCNQ top-deposition $(+\mathrm{F} 4)$ is marked by arrows. 
increase in HIB cannot be explained by photohole screening alone, as this effect is typically less than $0.4 \mathrm{eV}^{16,18,19} \mathrm{We}$ attribute it to substantial molecular conformation changes between the monolayer and multilayer; the chemical structure of TDATA offers substantially more degrees of freedom (compared to CBP) to assume three-dimensional conformations in the bulk. The adsorption on Au may force the molecule into a more planar conformation, which increases conjugation, thus lowering the energy gap and consequently the HIB. ${ }^{18}$ The deposition of $5 \AA$ F4-TCNQ on multilayer TDATA increased $\phi$ by $0.90 \mathrm{eV}$ and decreased the HIB by $0.65 \mathrm{eV}$. In addition, a substantial broadening of the TDATA features was observed.

The molecular levels of TDATA on Au precovered with 5 Å F4-TCNQ [Fig. 2(d)] were at significantly lower BEs compared to TDATA/Au, as expected (see above). The HIB was reduced to $\sim 0.00 \mathrm{eV}$ for monolayer TDATA/F4$\mathrm{TCNQ} / \mathrm{Au}$ and was $0.60 \mathrm{eV}$ for the multilayer film. $\phi$ decreased from 4.70 (4 ̊ TDATA/F4-TCNQ/Au) to $4.35 \mathrm{eV}$. The deposition of $5 \AA$ F4-TCNQ on top of $64 \AA$ TDATA/F4$\mathrm{TCNQ} / \mathrm{Au}$ increased $\phi$ again by $0.50 \mathrm{eV}$ and decreased the HIB by $0.25 \mathrm{eV}$. As in the previous experiment, the TDATA features were smeared out by top-deposition of F4-TCNQ.

It is remarkable that, for both CBP and TDATA, the spectral features and energy levels (HIB and sample work function) are essentially identical for both F4-TCNQ topdeposition and F4-TCNQ precovered Au substrates (Fig. 3). Since O/O CTC formation does not occur in F4-TCNQ/CBP the spectral shift for CBP/Au in Fig. 2(a) cannot be due to doping. The observed rigid shift, however, can be understood by the diffusion of F4-TCNQ through the CPB layer toward the Au substrate. F4-TCNQ then reacts with Au to form an $\mathrm{O} / \mathrm{M}$ CTC, which introduces an additional dipole $\mathrm{e}^{5}$ at the interface between the Au substrate and the CBP layer [Fig. 1(b)]. This dipole shifts the energy levels of the material on top toward $E_{F}$ and the sample work function increases, as is indeed observed in the experiment (Fig. 3). Analogous observations were made for F4-TCNQ deposited on top of tris(8-hydroxy-quinolino)aluminum $\left(\mathrm{Alq}_{3}\right)$ thin films (on pristine $\mathrm{Au}$ ), i.e., the valence features of $\mathrm{Alq}_{3}$ shifted toward $E_{F}$ by more than $0.5 \mathrm{eV}$ due to $\mathrm{F} 4-\mathrm{TCNQ}$ exposure. Increasing the initial $\mathrm{Alq}_{3}$ film thickness had the expected effect, i.e., the observed shift was smaller: ca. $0.2 \mathrm{eV}\left(100 \AA \mathrm{Alq}_{3}\right)$ and less than $0.1 \mathrm{eV}$ for a $150 \AA$ thick $\mathrm{Alq}_{3}$ film, since diffusion of F4-TCNQ toward the Au substrate becomes less efficient through the thicker organic layer. The F4-TCNQdiffusion scenario is fully congruent with the behavior of the spectra in Fig. 2(b). In this case, the Au substrate is saturated with F4-TCNQ molecules before CBP deposition. Therefore, the deposition of F4-TCNQ on top of CBP and the subsequent diffusion toward the $\mathrm{Au}$ substrate does not introduce any additional dipoles, and there is no energy shift or work function change due to F4-TCNQ deposition.

In essence, we can expect the same scenario to hold for the experiments done with TDATA. However, the valence spectrum of TDATA changes upon top-deposition of F4TCNQ, for $\mathrm{Au}$ as F4-TCNQ/Au substrates. An effective $p$-type doping in TDATA can now be invoked to explain the spectral shift for TDATA/Au toward $E_{F}$ after F4-TCNQ deposition in Fig. 2(c). However, the results presented in Fig. 2(d) indicate that the observed energy position of F4-TCNQ/ TDATA/Au may not be representative of $p$-type doping effects alone. The valence spectrum energy position and sample $\phi$ for F4-TCNQ/TDATA/F4-TCNQ/Au are identical to those of F4-TCNQ/TDATA/Au. This means that precovering the Au substrate with the acceptor, and thus the introduction of additional dipoles between Au and TDATA, did not result in any changes in the final sample properties. Consequently, part of the observed top-deposited F4-TCNQ induced shift in Fig. 2(c) may well be due to F4-TCNQ diffusing to the metal substrate, and changing the interface properties. Generally, it is difficult to disentangle the effects of doping and acceptor-modified substrate on the energy level positions, when the substrate interacts with the dopant.

In conclusion, the comparison of the energy levels measured for two organic heterosystems involving the acceptor F4-TCNQ highlighted that the issue of interdiffusion of small molecules through organic layers toward electrodes has to be taken into account when discussing the energy level alignment processes at $\mathrm{O} / \mathrm{M}$ and $\mathrm{O} / \mathrm{O}$ interfaces. We have observed energy level shifts toward $E_{F}$ induced by topdeposited F4-TCNQ due to diffusion of the acceptor toward the metal substrate and subsequent dipole layer formation (acceptor/metal charge transfer). This mechanism mimics $p$-type doping of the organic layer, even in the absence of CTC formation between the two organic materials.

This work was supported by the European Commission under Contract No. NMP-3-CT-2006-033197 ("ICONTROL") and the Sfb448 (DFG). N.K. acknowledges financial support by the Emmy-Noether-Program (DFG).

${ }^{1}$ K. Walzer, B. Maennig, M. Pfeiffer, and K. Leo, Chem. Rev. (Washington, D.C.) 107, 1233 (2007).

${ }^{2}$ M. Gross, D. C. Müller, H.-G. Nothofer, U. Scherf, D. Neher, C. Bräuchle, and K. Meerholz, Nature (London) 405, 661 (2000).

${ }^{3}$ J. Blochwitz, M. Pfeiffer, T. Fritz, and K. Leo, Appl. Phys. Lett. 73, 729 (1998).

${ }^{4}$ W. Gao and A. Kahn, Appl. Phys. Lett. 82, 4815 (2003).

${ }^{5}$ N. Koch, S. Duhm, J. P. Rabe, A. Vollmer, and R. L. Johnson, Phys. Rev. Lett. 95, 237601 (2005).

${ }^{6}$ S. Duhm, H. Glowatzki, J. P. Rabe, N. Koch, and R. L. Johnson, Appl. Phys. Lett. 90, 122113 (2007).

${ }^{7}$ L. Romaner, G. Heimel, J.-L. Brédas, A. Gerlach, F. Schreiber, R. L. Johnson, J. Zegenhagen, S. Duhm, N. Koch, and E. Zojer, Phys. Rev. Lett. 99, 256801 (2007).

${ }^{8}$ W. Gao and A. Kahn, J. Appl. Phys. 94, 359 (2003).

${ }^{9}$ X. Zhou, M. Pfeiffer, J. Blochwitz, A. Werner, A. Nollau, T. Fritz, and K. Leo, Appl. Phys. Lett. 78, 410 (2001).

${ }^{10}$ X. Zhou, J. Blochwitz, M. Pfeiffer, A. Nollau, T. Fritz, and K. Leo, Adv. Funct. Mater. 11, 310 (2001).

${ }^{11}$ I. G. Hill, A. Rajagopal, and A. Kahn, J. Appl. Phys. 84, 3236 (1998).

${ }^{12}$ Y. Shirota, J. Mater. Chem. 15, 75 (2005).

${ }^{13}$ R. L. Johnson and J. Reichardt, Nucl. Instrum. Methods Phys. Res. 208, 791 (1983).

${ }^{14}$ M. Meneghetti and C. Pecile, J. Chem. Phys. 84, 4149 (1986).

${ }^{15}$ J. S. Chappell, A. N. Bloch, W. A. Bryden, M. Maxfield, T. O. Poehler, and D. O. Cowan, J. Am. Chem. Soc. 103, 2442 (1981).

${ }^{16}$ A. Kahn, N. Koch, and W. Y. Gao, J. Polym. Sci., Part B: Polym. Phys. 41, 2529 (2003).

${ }^{17}$ H. Ishii, K. Sugiyama, E. Ito, and K. Seki, Adv. Mater. 11, 605 (1999).

${ }^{18}$ N. Koch, G. Heimel, J. Wu, E. Zojer, R. L. Johnson, J.-L. Brédas, K. Müllen, and J. P. Rabe, Chem. Phys. Lett. 413, 390 (2005).

${ }^{19}$ I. G. Hill, A. J. Mäkinen, and Z. H. Kafafi, J. Appl. Phys. 88, 889 (2000) 